\title{
COVID-19 pandemic: what consequences for cardiac rehabilitation?
}

\author{
Carlo Vigorito ${ }^{1}$, Pompilio Faggiano ${ }^{2}$, Gian Francesco Mureddu ${ }^{3}$ \\ ${ }^{1}$ University of Naples Federico II; ${ }^{2}$ Spedali Civili, Brescia; ${ }^{3}$ S. Giovanni-Addolorata Hospital, Rome, Italy
}

The ongoing COVID-19 pandemic spreading all around the world has stressed over its capabilities and determined profound changes in the health systems in all countries and has caused hundreds of thousand deaths. Health professionals have been called to a tremendous effort to deal with this emergency, often contaminating or succumbing themselves to the disease.

Patients with pre-existing cardiovascular disease infected with COVID-19 have an increased risk of severe disease and worse outcome, including death. Patients with COVID-19 infections have profound involvement not only of the pulmonary system, but also variegate involvement of other organs (multi organ failure, MOF), particularly of cardiovascular system (mainly through acute hypoxic myocardial injury, myocarditis, arterial and venous thromboembolism) [1]. Intensive or sub-intensive care units have carried the greatest load, but several hospital wards have been reconverted to COVID units, particularly internal medicine or specialties, like cardiology or pneumology, to face the growing wave of the disease.

This revolutionary transformation has had a strong implication for cardiology. Many cardiology units or Coronary Care Units (CCU) have been downsized and induced to allocate dedicated space and personnel to emergency COVID units or ICU. But COVID-19 storm has also had a deep impact for cardiac rehabilitation (CR): some CR units have been closed and medical or nurse staff moved to COVID units, others have seen a reduction of beds or staff, thus limiting their operative capabilities. To understand how Italian CR units are being re-modulated in this pandemia, the Italian Association of Cardiac Prevention and Rehabilitation (IACPR) has launched an online survey on the impact of COVID-19 on their activity, whose results will be the basis for prospecting different actions at national level and to understand the best way to reorganize the activities during and after the storm of the disease [2]. COVID-19 has had profound implications on the organization of $\mathrm{CR}$ also in other European countries, in many cases reducing or stopping $\mathrm{CR}$ programs (Hansen D, and Wilhelm M, personal communications).

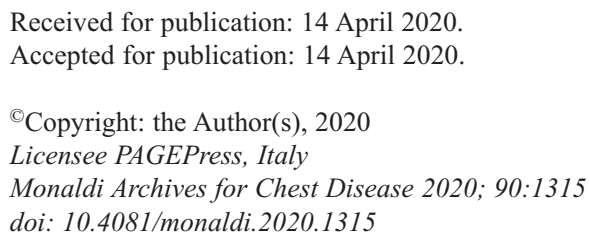

This article is distributed under the terms of the Creative Commons Attribution Noncommercial License (by-nc 4.0) which permits any noncommercial use, distribution, and reproduction in any medium, provided the original author(s) and source are credited.
Still operative CR units have to adapt their organization and programs according to the local health systems and to the different burden of the disease, with many differences among Italian regions. IACPR has issued general recommendations for CR activities during COVID-19 pandemia [3]. In general, active CR centers have seen in these days a reduced referral to residential CR from the traditional prevalent source (cardiac surgery, PTCA or other planned interventions usually referred to CR such as Pacemaker, ICD/CRT implantations), since all these elective activities have been postponed in large part. As far as Phase 2 outpatients CR, many elective in-hospital activities or outpatient sessions or visits have been postponed in the last 2 months. In addition, reduction of ACS admissions to CCU has occurred, since many patients refuse hospitalization or do not reach the hospital in time, or die at home. While the total admission to CCU has been largely reduced, the proportion of STEMI vs NSTEMI has increased, indicating that only the worse ACS patients, those with worse symptoms, are reaching the hospital, while many patients with fewer symptoms prefer to stay at home. Similarly, many heart failure (HF) patients avoid hospital admission even if the clinical status is worsening. However, In those patients who are referred and admitted to CR, the organization, provision and delivery of rehabilitative programs must be remodulated in COVID-19 era, according to the recommendations recently issued by the CR section of European Association of Preventive Cardiology (EAPC) [4]. The more relevant of these recommendations are establishing priorities for referral to $\mathrm{CR}$, increase patients turnover, adopt appropriate precautions during training (masks, distancing, avoid group exercises), shorten the programs, keep track of discharged patients and follow them with remote assessment.

In the medium time scenario, the challenge for CR units will be to be prepared to face for some time a population of patients at higher cardiovascular risk than usual: this will be composed of patients with ACS (STEMI or NSTEMI) or with worsening HF not hospitalized for the acute event, due to missed diagnosis or patient refusal. Many of these patients may have had reduced out of hospital attention from the practitioner or the specialist, which at this time are mostly focused on COVID-19 treatment, leading to inadequate secondary prevention, with poor drug adherence and traditional risk factors control, thus allowing progression of underlying cardiovascular disease. In addition, many ACS patients may have had a late admission to CCU due temporary shortage of ambulance availability, leading to delayed or unsuccessful revascularization. Occasionally, some patients are having systemic thrombolysis, rather than percutaneous revascularization. Accordingly, all these circumstances may most likely lead to the growth of a ACS population resembling that of pre-PTCA era, with more frequent STEMI, left ventricular systolic dysfunction and/or $\mathrm{HF}$, poorer physical function for longer inactivity and poorer prognosis. CR strategies and programs should therefore be tailored on the peculiarities of these patients, who are at higher risk and with the majority of them being elderly and often frail. 
In addition, beyond these organizative aspects, there are other implications of this pandemic on CR. First of all, CR units should develop strategies on how to handle cardiac patients who have developed COVID-19 infection during admission in medical or surgical units for ACS, HF, cardiac surgery, etc., and thereafter discharged and referred to CR. Of course, strict rules should be established for these referrals, that should be reserved only to infected patients after COVID-19 swabs are negative and chest imaging recovered, and to those at higher risk, who need residential CR for stabilization of an acute coronary event and for whom immediate return at home may be dangerous. Of interest, while the acute cardiovascular complications of COVID-19 infection are recognized, its medium to long term consequences on cardiovascular recovery after an acute event are still largely unknown. Coronavirus infection may largely affect cardiovascular and other systems, and these aspects may complicate the clinical handling and even the modalities of rehabilitative programs or drug strategies in these patients. A closer link with other specialists (mainly lung and thrombosis specialists) will allow to face up to these unprecedented clinical scenarios.

Another side of the coin is how to deal with patients, unlikely but possible, developing COVID-19 infection during residential CR. In this setting, CR Units should be prepared to implement all protections for health professionals, patients, contact and relatives, including isolating the infected, stopping rehabilitative program, and to make difficult choices on the best discharge destination (home quarantine, cardiology or COVID wards,) depending from the more severe cardiologic or pneumologic clinical damage.

In summary, COVID-19 infection outbreak is having, and will likely have for a long period, a profound impact on CR organization and provision of $\mathrm{CR}$ programs. Cardiology and Cardiac Rehabilitation scientific societies should be prepared to face this new challenge, particularly by re-educating cardiac patients to refer to cardiologic services in case of worsening of the underlying disease, and reinforcing the need of maintaining secondary prevention despite all difficulties, in order to reduce as much as possible the negative functional and prognostic impact of COVID19 infection in cardiovascular patients.

\section{References}

1. Huang C, Wang Y, Li X, et al. Clinical features of patients infected with 2019 novel coronavirus in Wuhan, China. Lancet 2020;395:497-506.

2. Italian Association for Cardiovascular Prevention and Rehabilitation (AICPR). Survey on the impact of the Covid-19 pandemic on the activities of the Cardiac Rehabilitation Centers in Italy. Available from: https://forms.office.com/Pages/Response Page.aspx?id=bID-cE2T2E2SHNVefPPPiHDQ81J5XqVOnn KORzuh9WdUMFNQQIVBTIlBQlpYMUIRUTJBWUQ4WDk zWC4u

3. Italian Association for Cardiovascular Prevention and Rehabilitation (AICPR). [Raccomandazioni AICPR per le attività di cardiologia riabilitativa e preventiva nella fase di emergenza pandemica da COVID-19].[in Italian]. Available from: http:// www.iacpr.it/uploaded_images/areacovid/RACCOMANDAZIO NI_AICPR PER LE ATTIVITA DI CARDIOLOGIA.pdf

4. European Society of Cardiology [Internet]. Recommendations on how to provide cardiac rehabilitation activities during the COVID-19 pandemic. Released by: Secondary Prevention and Rehabilitation Section of the European Association of Preventive Cardiology (EAPC), April 7, 2020. Available from: https:// www.escardio.org/Education/Practice-Tools/CVD-preventiontoolbox/recommendations-on-how-to-provide-cardiac-rehabi litation-activities-during-the-c 